\title{
LA AMPLIACIÓN DE LA PLATAFORMA CONTINENTAL EN EL ATLÁNTICO SUR: EL ENFRENTAMIENTO MARÍTIMO ENTRE ARGENTINA Y REINO UNIDO
}

\author{
THE EXTENSION OF THE CONTINENTAL PLATFORM IN THE \\ SOUTH ATLANTIC: THE MARITIME CONFLICT BETWEEN \\ ARGENTINA AND THE UNITED KINGDOM
}

\author{
ÁNGELES JIMÉNEZ GARCÍA-CARRIAZO*
}

Recibido: 02/02/2017

Aceptado: 08/03/2017

\begin{abstract}
SUMARIO: I.- INTRODUCCIÓN. II.- LOCALIZACIÓN E HISTORIA. III.- LOS ESPACIOS MARINOS DE LAS ISLAS MALVINAS, LAS ISLAS GEORGIAS DEL SUR Y LAS ISLAS SÁNDWICH DEL SUR. IV. PROCEDIMIENTO ANTE LA COMISIÓN DE LÍMITES DE LA PLATAFORMA CONTINENTAL. V. EL TRATADO DE LA ANTÁRTIDA. VI. LAS RECOMENDACIONES DE LA COMISIÓN DE LÍMITES DE LA PLATAFORMA CONTINENTAL. VII. LA DELIMITACIÓN DE LA PLATAFORMA CONTINENTAL AMPLIADA Y SU RELACIÓN CON LA DETERMINACIÓN DEL LÍMITE EXTERIOR. VIII. LA PLATAFORMA CONTINENTAL DE OTROS TERRITORIOS DISPUTADOS O NO AUTÓNOMOS. IX. CONCLUSIÓN. X. BIBLIOGRAFÍA.
\end{abstract}

\begin{abstract}
SUMMARY: I- INTRODUCTION. II.- LOCATION AND HISTORY. III.- FALKLAND ISLANDS, SOUTH GEORGIA AND SANDWICH ISLANDS' MARINE AREAS. IV. PROCEDURE BEFORE THE COMMISSION ON THE LIMITS OF THE CONTINENTAL SHELF. V. THE ANTARCTIC TREATY. VI. THE COMMISSION ON THE LIMITS OF THE CONTINENTAL SHELF'S RECOMMENDATIONS. VII. DELIMITATION OF THE EXTENDED CONTINENTAL SHELF AND ITS RELATIONSHIP WITH THE ESTABLISHMENT OF THE OUTER LIMIT. VIII. OTHER DISPUTED OR NON-SELF-GOVERNING TERRITORIES' CONTINENTAL SHELF. IX. CONCLUSION. X. BIBLIOGRAPHY.
\end{abstract}

Resumen: La histórica controversia entre Argentina y Reino Unido por los territorios en el Atlántico Sur ha sido estudiada durante décadas desde una perspectiva territorial. Sin embargo, la posibilidad contemplada por la CNUDM de ampliar la plataforma continental más allá de las $200 \mathrm{~mm}$ desplaza esta diferencia de soberanía terrestre al mar. La explotación de la plataforma continental permite el acceso del Estado ribereño a nuevos recursos naturales, así como la consolidación de su presencia en la escena internacional. Gracias a la ampliación, el Estado disfrutará de derechos soberanos de exploración y explotación sobre el lecho y el subsuelo de esta área. Argentina y Reino Unido, conscientes de los intereses en juego, han incluido los territorios disputados en sus respectivas presentaciones a la Comisión de Límites. Sin embargo, este organismo no está facultado para decidir sobre la soberanía, sus recomendaciones no pueden ser consideradas como un instrumento de arreglo de controversias.

\footnotetext{
* Doctorando de Derecho Internacional Público de la Universidad de Jaén. Número ORCID: 0000-00030557-8322. Correo electrónico: angeles.jgcarriazo@gmail.com.
} 
Palabras clave: Derecho del mar - Plataforma continental ampliada - Comisión de Límites - Islas Malvinas - Antártida.

Abstract: The landmark dispute between Argentina and the United Kingdom over the territories in the South Atlantic has been studied for decades from a territorial perspective. However, the possibility provided for under UNCLOS to extend the continental shelf beyond $200 \mathrm{~mm}$ shifts this land sovereignty dispute to the sea. The exploitation of the continental shelf allows the access of the coastal State to new natural resources, as well as the strengthening of its presence on the international arena. Thanks to the extension, the State will enjoy sovereign rights for the purpose of exploring and exploiting the seabed and subsoil of this area. Argentina and the United Kingdom, aware of the interests at stake, have included the disputed territories in their respective submissions to the Commission on the Limits. However, this body is not entitled to rule on sovereignty, its recommendations cannot be considered as a mean of dispute settlement.

Key words: Law of the sea - Extended continental shelf - Commission on the Limits Falkland Islands - Antarctica.

En marzo de 2016, la Comisión de Límites de la Plataforma Continental (en adelante, CLPC o Comisión) emitió sus recomendaciones acerca de la solicitud de ampliación de la plataforma continental argentina. Este asunto pone de nuevo sobre la mesa la controversia entre Reino Unido y Argentina acerca de la soberanía sobre las Islas Malvinas, Georgias del Sur y Sándwich del Sur, así como la determinación de sus respectivos espacios marinos.

\section{I.- INTRODUCCIÓN}

Para comprender el verdadero alcance de la cuestión planteada entre ambos Estados conviene tomar en consideración el modo en el que el Derecho del Mar aborda el tema de la plataforma continental ampliada.

La plataforma continental es la prolongación submarina del territorio. Los Estados ribereños ostentan derechos de soberanía a los efectos de la exploración y la explotación de los recursos naturales del lecho y el subsuelo que conforman sus respectivas plataformas continentales. Estas facultades se ejercen hasta el borde exterior del margen continental, o bien hasta una distancia de 200 millas marinas contadas desde las líneas de base a partir de las cuales se mide la anchura del mar territorial en los casos en que el borde exterior del margen continental no llegue a esa distancia ${ }^{1}$.

La exploración de los océanos y la explotación intensiva de los recursos marinos se realiza principalmente con fines económicos. Sin embargo, el reconocimiento de estos derechos también ha despertado las ambiciones de soberanía de los Estados ribereños ante la posibilidad que ofrece la Convención de Naciones Unidas sobre el Derecho del Mar de 1982 (en adelante, Convención de Montego Bay o Convención) de ampliar sus plataformas continentales hasta las 350 millas marinas contadas desde las líneas de base, o de 100 millas marinas a partir de la isóbata de los 2.500 metros de profundidad ${ }^{2}$.

\footnotetext{
${ }^{1}$ Artículos 76.1 y 77 de la Convención de Montego Bay.

${ }^{2}$ Artículo 76.5 de la Convención de Montego Bay. 
La figura de la plataforma continental ampliada se ha convertido, por tanto, en un nuevo escenario de tensiones políticas en el que los conflictos territoriales previos se trasladan a un espacio marítimo más extenso.

En los procedimientos de ampliación de la plataforma continental, la CLPC se encuentra con títulos de jurisdicción superpuestos, reivindicaciones de soberanía o conflictos pendientes. Sin embargo, debe dejar de lado toda controversia territorial o marítima y adoptar las decisiones sin prejuzgar las cuestiones relativas a la fijación de los límites entre Estados ${ }^{3}$.

En este contexto nos encontramos con el caso de las Islas Malvinas, Georgias del Sur y las Islas Sándwich del Sur, tres archipiélagos administrados de facto por Reino Unido y reivindicados por Argentina. La conocida fricción por estos territorios insulares se ha convertido en una nueva controversia de carácter marítimo, cuya resolución puede marcar el devenir de la plataforma antártica ${ }^{4}$.

\section{II.- LOCALIZACIÓN E HISTORIA}

Con una superficie de unos $12.000 \mathrm{~km}^{2}$, el archipiélago de las Malvinas ${ }^{5}$ lo componen más de doscientas islas situadas en la plataforma continental sudamericana, a 464 kilómetros del litoral argentino.

Aunque el primer avistamiento se lo disputan españoles, holandeses y británicos, fue el navegante francés Louis Antoine de Bougainville quien fundó el primer asentamiento en Isla Soledad en 1764. Un año después, Reino Unido decidió emprender una expedición a las Islas Malvinas y estableció una base provisional en la Isla Trinidad ${ }^{6}$. En 1767, España reclamó a Francia el asentamiento fundado por Bougainville y la posesión del archipiélago le fue restituida.

Pese a la presencia británica en las islas y las sucesivas crisis diplomáticas, las Malvinas quedaron sujetas a gobernación española hasta 1811. En 1820, Argentina envió una fragata para reafirmar sus derechos sobre las Islas Malvinas esgrimiendo el principio uti possidetis.

Reino Unido ocupó el archipiélago en 1833, lo renombró como Islas Falkland y lo administró de manera ininterrumpida hasta 1982, año en el que Argentina invadió las islas. Los británicos recuperaron el control tras 73 días de guerra y restablecieron su administración ${ }^{7}$.

\footnotetext{
${ }^{3}$ Artículo 46 y Anexo 1 del Reglamento de la Comisión de Límites de la Plataforma Continental, CLCS/40/Rev.1.

${ }^{4}$ A pesar de la existencia del Tratado de la Antártida de 1959.

${ }^{5}$ Emplearemos la denominación española, Islas Malvinas, en todo caso con la excepción de aquellas situaciones en la que nos encontremos ante pronunciamientos británicos, para los que mantendremos la fórmula Islas Falkland.

${ }_{7}^{6}$ Isla Saunders en inglés.

${ }^{7}$ Sobre la discusión acerca de los títulos de soberanía esgrimidos por cada una de las partes Vid. FARAMIÑÁN GILBERT, J.M.; Situación actual del contencioso Malvinas (Falkland), Revista Española de Derecho Internacional, $\mathrm{n}^{\circ}$ 1-1985, Consejo Superior de Investigaciones Científicas, Instituto de Ciencias Jurídicas, Madrid, 1985, p. 135-151.
} 
A día de hoy, Argentina no acepta esta situación. Es más, en el instrumento de ratificación de la Convención de Montego Bay, el Gobierno argentino realizó una declaración afirmando "su soberanía legítima e inalienable sobre las Malvinas, Georgias del Sur, las Islas Sándwich del Sur, y sus espacios marinos respectivos”"

Actualmente, Argentina incluye a las Islas Malvinas en el Departamento Islas del Atlántico Sur de la Provincia de Tierra del Fuego, Antártida e Islas del Atlántico Sur.

Por otro lado, el denominado territorio británico de ultramar de las Islas Georgia del Sur y Sándwich del Sur lo conforman dos archipiélagos australes administrados por Reino Unido desde el siglo XIX y cuya soberanía también reclama Argentina.

Estas islas se encuentran en el Arco de las Antillas Australes del océano Atlántico Suroccidental, justo encima del continente antártico. El archipiélago de las Georgias del Sur lo conforman una isla principal y varias pequeñas que la rodean; el archipiélago de las Sándwich del Sur lo compone una cadena de 390 kilómetros de largo de once pequeñas islas de origen volcánico.

Originalmente deshabitadas, actualmente Georgia del Sur cuenta con una expedición científica de la British Antarctic Survey que vive en la isla de manera permanente.

El primer desembarco conocido en estas islas lo realizó el capitán James Cook en $1775^{9}$, quien cartografió la zona y reclamó las islas para la Corona británica. En 1908, Reino Unido anexionó las islas, considerándolas como Dependencias de las Islas Malvinas. La carta de patente emitida por el gobierno británico el 21 de julio de dicho año ${ }^{10}$ declaraba que:

"el grupo de islas conocido como Georgias del Sur, Orcadas del Sur, Shetlands del Sur y Sándwich del Sur y el territorio conocido como Tierra de Graham, situados en el Océano Atlántico Sur, al sur del paralelo 53 de latitud sur y comprendidos entre los $20^{\circ}$ y $80^{\circ}$ de longitud oeste, forman parte de nuestros Dominios y conviene que se tomen disposiciones para su gobierno como Dependencias de nuestra Colonia de las Falklands”.

En 1927 se inició la controversia por este territorio. Argentina formuló sus pretensiones de soberanía sobre las Georgias del Sur, ampliando posteriormente la reclamación a todos los territorios de las Dependencias de las Islas Malvinas, incluidas las Islas Sándwich del Sur. Dicho Estado consideraba que había sido el primer país en ocupar las Georgias del Sur en 1904, cuando la Compañía Argentina de Pesca S.A. se instaló permanentemente en ella. Actualmente, Argentina también incluye los dos archipiélagos

\footnotetext{
${ }^{8}$ Declaración de Argentina de 1 de diciembre de 1995: “The Argentine Republic reaffirms its legitimate and inalienable sovereignty over the Malvinas and the South Georgia and South Sandwich islands and their respective maritime and island zones, which form an integral part of its national territory".

${ }^{9}$ Existen referencias a que el comerciante inglés Anthony de la Roche avistó las islas en 1675. Asimismo, el capitán español Gregorio Jerez documentó el avistamiento en 1756 y bautizó al archipiélago como las Islas de San Pedro. Vid. SÁNCHEZ, R.; Antártida: Introducción a un continente remoto, Editorial Albatros, 2008, p. 100.

${ }^{10}$ Vid. Extracto de British and Foreign State Papers, 1907-08, Vol. 101, 1912, p. 76-77: "the group of islands known as South Georgia, the South Orkneys, the South Shetlands, and the Sandwich Islands, and the territory known as Graham's Land, situated in the South Atlantic Ocean to the south of the 53th parallel of south latitude, and lying between the 20th and the 80th degrees of west longitude, are part of our Dominions, and it is expedient that provision should be made for their government as Dependencies of our Colony of the Falklands". 
en el Departamento Islas del Atlántico Sur de la Provincia de Tierra del Fuego, Antártida e Islas del Atlántico Sur.

Pese a lo anteriormente relatado, Reino Unido ha ejercido el control de facto sobre ambos archipiélagos desde 1908, con la excepción de un breve período durante la Guerra de las Malvinas en 1982, cuando las islas fueron parcialmente controladas por Argentina.

Una vez localizado el conflicto, no debemos olvidar que la resolución 2065 de 16 de diciembre de 1965 de la Asamblea General de Naciones Unidas reconoció la existencia de una disputa de soberanía entre el Reino Unido y Argentina en relación con las Islas Malvinas. Asimismo, afirmó el estatus de territorio no autónomo e instó a las partes a resolver la situación colonial.

\section{III.- LOS ESPACIOS MARINOS DE LAS ISLAS MALVINAS, LAS ISLAS GEORGIAS DEL SUR Y LAS ISLAS SÁNDWICH DEL SUR}

Antes de adentrarnos en el estudio en profundidad de la plataforma continental ampliada de estos archipiélagos y con el fin de tener una visión completa de la cuestión, analizaremos brevemente sus espacios marinos, las definiciones que respectivamente han realizado Argentina y Reino Unido, así como los acuerdos que ocasionalmente han alcanzado.

\section{Líneas de base y mar territorial ${ }^{11}$}

Tanto Reino Unido como Argentina han definido las líneas de base de las Islas Malvinas, pero utilizando diferentes puntos para el trazado, por lo que el resultado final tiene distinto alcance.

En 1989, el Parlamento británico promulgó sus órdenes sobre el mar territorial de las Islas Malvinas, las Georgias del Sur y las Islas Sándwich del Sur ${ }^{12}$ que establecen que: "la línea de base desde la cual se mide el mar territorial adyacente [...] será la línea de bajamar a lo largo de la costa de todas las islas”13. Dos años más tarde, Argentina establecía sus líneas de base en su Ley de Espacios Marítimos argentinos ${ }^{14}$, empleando líneas de base normal y de base recta. Cada Estado utiliza sus respectivas líneas de base para establecer el mar territorial hasta las 12 millas marinas.

Pese a la similitud de las declaraciones, Argentina afrontó su tarea con más minuciosidad, así lo corroboran los 108 puntos que nominó para el trazado de las líneas

\footnotetext{
${ }^{11}$ Las líneas de base son las líneas a partir de las cuales se miden los espacios marinos. El mar territorial es el espacio que se extiende hasta las 12 millas marinas y sobre el que el Estado ribereño ejerce plena soberanía.

12 The Falkland Islands (Territorial Sea) Order 1989 (No. 1993 of 1989) dated $1^{\text {st }}$ November 1989: "the baseline from which the breadth of the territorial sea adjacent to the Falkland Islands is measured shall be the low-water line along the coast of all islands”. The South Georgia and South Sandwich Islands (Territorial Sea) Order 1989 (1989 No. 1995): "the baseline from which the breadth of the territorial sea adjacent to South Georgia and South Sandwich Islands is measured shall be the low-water line along the coast of all islands”.

${ }^{13}$ Con la excepción en el caso de las Malvinas de la línea de base entre el Cabo Carysfort, el Cabo Percival y el Cabo MacBride, para la que se emplean líneas loxodrómicas.

${ }^{14}$ Artículo 1 y Anexo I de la Ley no 23.968 de Espacios marítimos argentinos, de 10 de septiembre.
}

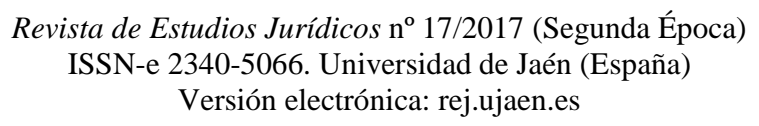


de base de las Malvinas, frente a los 22 que emplearon los británicos. Como consecuencia, el Estrecho de San Carlos (Falkland Sound) queda incluido en las aguas internas según el diseño de Reino Unido, pero no en las argentinas ${ }^{15}$.

\section{Zona contigua ${ }^{16}$}

Argentina ha establecido una zona contigua que se extiende hasta las 24 millas marinas contadas desde las líneas de base ${ }^{17}$.

\section{Zona económica exclusiva / Zona de pesca ${ }^{18}$}

En 1986, el gobernador británico de las Malvinas, aludiendo a que el Derecho Internacional permitía delimitar zonas de pesca hasta las 200 millas marinas ${ }^{19}$, estableció una zona provisional de conservación y gestión de las pesquerías para el archipiélago malvinense de un radio de 150 millas marinas, excepto en una pequeña porción al suroeste donde el límite lo marcaba una línea loxodrómica ${ }^{20}$. Algunos autores han visto en este dato un reconocimiento al derecho de Argentina a declarar su zona económica exclusiva ${ }^{21}$.

En 1990, Argentina y Reino Unido acordaron cooperar en la protección de las reservas pesqueras existentes en el Atlántico Sur en la zona comprendida entre los $45^{\circ}$ y $60^{\circ}$ de latitud sur a través de la Declaración Conjunta sobre la Conservación de Recursos Pesqueros $^{22}$. En consecuencia, Reino Unido extendió el límite de la zona de pesca hasta las 200 millas marinas ${ }^{23}$.

15 Vid. AMSTRONG P.; FORBES, V.; The Falkland Islands and their Adjacent Maritime Area, International Boundaries Research Unit, Maritime Briefing, Durham University, 1997, vol. 2 nº 3.

${ }^{16}$ La zona contigua es el espacio adyacente al mar territorial en la cual el Estado puede prevenir y perseguir la inobservancia de su normativa aduanera, fiscal, de inmigración y sanitaria que se cometa en su territorio o en su mar territorial. La zona contigua abarca desde las 12 hasta las 24 millas marinas.

${ }^{17}$ Artículo 4 de la Ley n 23.968 de Espacios marítimos argentinos, de 10 de septiembre de 1991.

${ }^{18}$ La zona económica exclusiva es una franja marítima que se extiende desde el límite exterior del mar territorial hasta una distancia de 200 millas marinas. El Estado ejerce derechos de soberanía y jurisdicción sobre todas las actividades relacionadas con los recursos naturales, las islas artificiales, la investigación científica y la protección del medio marino. La zona de pesca es una zona análoga que equivaldría a una subcategoría de la zona económica exclusiva.

${ }^{19}$ Proclamation No. 4 of 1986 Interim Fishery Conservation and Management Zone dated 29 October: "Whereas the Falkland Islands are entitled under international law to a fisheries limit of 200 nautical miles from the baselines from which the breadth of territorial seas is measured subject to the boundary with a neighbouring state prescribed by the rules of international law concerning the delimitation of maritime jurisdiction".

Esta declaración está en línea con la evolución del Derecho del Mar de la época, y especialmente, con la formulación del artículo 57 CNUDM, si bien es cierto que la Convención aún no había entrado en vigor.

${ }^{20}$ Ibid.: "The zone will have as its inner boundary the outer limits of the territorial sea of the Falkland Islands and has as itis seaward boundary the line formed by the circumference of a circle which has a radius of 150 nautical miles and its centre at latitude $51^{\circ} 40^{\prime} \mathrm{S}$, longitude $59^{\circ} 30^{\prime} \mathrm{W}$, except that between the points on that circumference situated at latitude $52^{\circ} 30^{\prime} \mathrm{S}$, longitude $63^{\prime} 19.25^{\prime} \mathrm{W}$ and latitude $54^{\circ}$ $08.68^{\prime} \mathrm{S}$, longitude $60^{\circ} 00^{\prime} \mathrm{W}$ the seaward boundary shall be a rhumb line".

${ }^{21}$ Vid. AMSTRONG, P.; Falklands Oil, IBRU Boundary and Security Bulletin, Durham University, julio 1994.

22 Declaración conjunta sobre la conservación de recursos pesqueros de los Gobiernos de la República Argentina y el Reino Unido de Gran Bretaña de 28 de noviembre de 1990.

${ }^{23}$ Proclamation No. 2 of 1990 dated 20 December (amended by Proclamation No. 1 of 1994): “a line drawn anti-clockwise 200 nautical miles from the nearest points on the baseline of the territorial sea of the Falkland Islands". 
Respecto a las Georgias del Sur y las Islas Sándwich del Sur, la Declaración británica de $1993^{24}$ estableció una zona marítima que se extiende hasta las 200 millas marinas y en la que los derechos a ejercer sobre las aguas se confieren a la Corona británica.

En lo relativo a Argentina, la ya mencionada Ley de Espacios Marítimos ${ }^{25}$ proclamó la existencia de una zona económica exclusiva con una extensión de 200 millas marinas que aplica a la totalidad del territorio argentino (y, por ende, a las islas en cuestión).

Conviene destacar que dicho Estado declaró en 1966 la existencia de una figura que presenta cierta similitud con la zona económica exclusiva. Mediante la Ley n ${ }^{\circ} 17.094^{26}$, Argentina extendió su soberanía al mar adyacente a su territorio hasta una distancia de 200 millas marinas.

\section{Plataforma continental ${ }^{27}$}

En 1991, Argentina recoge la figura de la plataforma continental en su legislación ${ }^{28}$ de conformidad con la formulación definitiva del artículo 76 de la Convención de Montego Bay.

Al igual que ocurría con la zona económica exclusiva, la Ley $\mathrm{n}^{\circ} 17.094$ había incorporado al ordenamiento jurídico argentino una figura análoga a la plataforma continental. El artículo 2 de dicha ley preveía la extensión de la soberanía argentina al lecho del mar y al subsuelo de las zonas submarinas adyacentes a su territorio hasta una profundidad de 200 metros o más allá de este límite, hasta donde la profundidad de las aguas suprayacentes permitiesen la explotación de los recursos naturales de dichas zonas.

En el mismo año, Reino Unido concretó su regulación sobre la plataforma continental de las Islas Malvinas. El poder legislativo británico confería a la Corona todos los derechos que pudiesen ejercerse sobre el lecho y el subsuelo de la plataforma

\footnotetext{
${ }^{24}$ Proclamation (Maritime Zone) No. 1 of 1993 dated 7 May 1993.

${ }^{25}$ Artículo 5 de la Ley no 23.968 de Espacios marítimos argentinos, de 10 de septiembre de 1991.

${ }^{26}$ Ley n 17.094 de extensión del mar argentino hasta 200 millas de 29 de diciembre de 1966. Esta ley se inscribe en la oleada de reivindicaciones de soberanía que se produjeron en Latinoamérica después de la Declaración Truman.

Desde hacía cincuenta años, ya se venían produciendo avances en Argentina en este ámbito. En 1916, Segundo Storni elaboró una doctrina de reivindicación de la plataforma continental, pensando no sólo en la pesca sino en todos los recursos. En 1944 se declaró una zona de reserva minera sobre la plataforma continental, pero sin precisiones de mayor alcance. En 1946, a través del decreto 14.708 se reivindicó la soberanía argentina sobre el mar epicontinental y el zócalo continental. Vid. ARMAS PFIRTER, F.; El límite exterior de la plataforma continental, Anuario Argentino de Derecho Internacional, X, Córdoba, Argentina, 2000, p. 247.

${ }^{27}$ La plataforma continental comprende el lecho y el subsuelo de las áreas submarinas que se extienden a lo largo de la prolongación natural de su territorio hasta el borde exterior del margen continental, o bien hasta una distancia de 200 millas marinas. Los Estados ribereños ostentas derechos de soberanía a los efectos de la exploración y la explotación de los recursos existentes en este espacio.

${ }^{28}$ Artículo 6 de la Ley no 23.968 de Espacios marítimos argentinos, de 10 de septiembre de 1991.

Conviene recordar que los derechos del Estado ribereño existen ipso facto y ab initio en relación con su plataforma continental. Sentencia de la CIJ de 20 de febrero de 1969 en el asunto Plataforma Continental del Mar del Norte (República Federal de Alemania/Dinamarca; República Federal de Alemania/Países Bajos). Vid. Sentencia de la CIJ de 20 de febrero de 1969 en el asunto Plataforma Continental del Mar del Norte.
} 
continental que rodea el archipiélago malvinense ${ }^{29}$. El mismo día, la administración británica de las Malvinas aprobó una ordenanza ${ }^{30}$ de acuerdo con la cual, se supeditaba la exploración de la plataforma continental a la concesión de una licencia.

Sobre los archipiélagos de Georgias del Sur y Sándwich del Sur aplica la anteriormente mencionada Declaración de $1993^{31}$, pues los derechos conferidos a la Corona británica también alcanzaban al lecho y subsuelo marino, así como a los recursos naturales depositados en esta área.

Tras consagrar la figura de la plataforma continental en sus respectivas legislaciones, ambos Estados firmaron una declaración tendente a alentar las actividades offshore ${ }^{32}$. El objetivo era favorecer la exploración y explotación de hidrocarburos en el Atlántico Suroccidental, y en particular, en la denominada "área especial”, situada entre el archipiélago de las Malvinas y Argentina, del lado malvinense de la línea de equidistancia. Estas disposiciones se adoptaron sin perjuicio de las reivindicaciones relativas a las Islas Malvinas, Georgias del Sur, Islas Sándwich del Sur y los espacios marítimos circundantes. Argentina denunció la declaración en $2007^{33}$ por las divergencias en la interpretación realizada por cada Estado.

\section{PROCEDIMIENTO ANTE LA COMISIÓN DE LÍMITES DE LA PLATAFORMA CONTINENTAL ${ }^{34}$}

Los Estados ribereños interesados en ampliar sus plataformas continentales más allá de las 200 millas marinas deben presentar a la CLPC suficientes argumentos geológicos y morfológicos que lo justifiquen. Para ello deben delimitar el borde exterior de la plataforma continental de conformidad con una compleja combinación de cuatro reglas - dos fórmulas y dos restricciones - que el artículo 76 de la Convención de Montego Bay recoge. Las Directrices científicas y técnicas de la CLPC ${ }^{35}$ pautan el procedimiento a seguir.

En primer lugar, el Estado ribereño debe ubicar el pie del talud continental que, salvo prueba en contrario, se entenderá como el punto de máximo cambio de gradiente en su base. Una vez localizado el pie del talud, se trazan las líneas de fórmulas, cuya

\footnotetext{
${ }^{29}$ Proclamation No. 1 of 1991 (Falkland Islands Continental Shelf) dated 22 November 1991: “Any rights exercisable over the seabed and subsoil of the continental shelf, including the natural resources thereof, beyond and adjacent to the territorial sea around the Falkland Islands are hereby vested in Her Majesty".

${ }^{30}$ Colony of the Falkland Islands, The Continental Shelf Ordinance 1991 ( $\mathrm{N}^{\mathrm{o}} 19$ of 1991).: "No person shall explore for any mineral in any designated area except under the authority of a licence granted".

${ }^{31}$ Proclamation (Maritime Zone) No. 1 of 1993 dated 7 May 1993.

32 Declaración Conjunta sobre Cooperación en Actividades Costa Afuera en el Atlántico Sudoccidental de 27 de septiembre de 1995.

${ }^{33}$ Carta del Ministro de Relaciones Exteriores de Argentina al Secretario de Asuntos Exteriores del Reino Unido de 27 de marzo de 2007.

${ }^{34}$ La CLPC es el órgano encargado de estudiar las reclamaciones con vistas a la extensión de los Estados ribereños más allá de 200 millas marinas. Se trata de un organismo autónomo compuesto por veintiún miembros, expertos en geología, geofísica e hidrografía, elegidos por los propios Estados Partes de la Convención.

La tarea de la Comisión es doble, pues, por un lado, realizan el examen de las solicitudes presentadas por los Estados ribereños y emiten sus correspondientes recomendaciones; y por otro, prestan asesoramiento científico y técnico dirigido a la formulación de las presentaciones

${ }^{35}$ Directrices científicas y técnicas de la Comisión de Límites de la Plataforma Continental aprobadas por la Comisión el 13 de mayo de 1999 en su quinto período de sesiones, Nueva York, 3 a 14 de mayo de 1999.

Revista de Estudios Jurídicos nº 17/2017 (Segunda Época)

ISSN-e 2340-5066. Universidad de Jaén (España)

Versión electrónica: rej.ujaen.es
} 
envolvente exterior se corresponde con el borde exterior de la plataforma continental. A las fórmulas se las conoce por el nombre de los geólogos que las propusieron:

- la “fórmula Gardiner” da como resultado una línea formada por puntos fijos localizados allá donde el espesor de los sedimentos es, al menos, el 1\% de la distancia más corta entre ese punto y el pie del talud;

- la “fórmula Hedberg” genera una línea trazada en relación con los puntos fijos situados a no más de 60 millas marinas del pie del talud continental.

Posteriormente, se evalúa la propuesta a través de la prueba de pertenencia, en virtud de la cual el Estado ribereño deberá demostrar que su plataforma continental se extiende más allá de las 200 millas marinas medidas desde las líneas de base.

Seguidamente, se aplican las líneas de restricciones, que impiden que la plataforma continental pueda ir más allá de las 350 millas marinas o de las 100 contadas desde la isóbata de 2.500 metros.

Finalmente, se procede a trazar los límites exteriores de la plataforma continental ampliada, atendiendo tanto al resultado de las líneas de fórmulas como al de las líneas de restricciones.

Así las cosas, la CLPC recibe la propuesta del Estado ribereño y los datos geológicos y geomórfológicos de apoyo. Tras el examen de la documentación facilitada, la Comisión emite unas recomendaciones, de acuerdo con las cuales los Estados ribereños establecerán los límites exteriores de su plataforma continental definitivos y obligatorios.

La Comisión no ostenta la autoridad legal para imponer el trazado del borde exterior del margen continental, sino que son los propios Estados ribereños los que establecen sus límites exteriores más allá de las 200 millas marinas. Así lo ha reconocido la sentencia del Tribunal Internacional del Derecho del Mar en el asunto Bangladesh/Myanmar ${ }^{36}$ :

“Del artículo 76.8 de la Convención se deduce claramente que los límites de la plataforma continental más allá de las 200 millas marinas sólo pueden ser establecidos por el Estado ribereño. Aunque se trata de un acto unilateral, la oponibilidad con respecto a otros Estados de los límites establecidos de esta manera depende de la satisfacción de los requisitos especificados en el artículo 76, en particular el cumplimiento por parte del Estado ribereño de la obligación de presentar a la Comisión información sobre los límites de la plataforma continental más allá de las 200 las millas marinas y la emisión por parte de la Comisión de recomendaciones relevantes en este sentido. Sólo una vez establecidos los límites por el Estado ribereño sobre la base de las recomendaciones de la Comisión, estos límites se convierten en «definitivos y obligatorios»".

\footnotetext{
${ }^{36}$ Sentencia del TIDM de 14 de marzo de 2012 relativo a la Controversia sobre la Delimitación de las Fronteras Marítimas entre Bangladesh y Myanmar (Bangladesh contra Myanmar), caso $n^{\circ} 16$, párrafo 407.
} 
Como ya se ha mencionado, en el caso de presentaciones en las que concurran controversias territoriales o marítimas, las decisiones de la Comisión no prejuzgarán cuestiones relativas a la fijación de los límites entre Estados.

En $2001^{37}$, Argentina y Reino Unido consideraron la posibilidad de coordinar las actividades preparatorias de las respectivas presentaciones en el área de Malvinas, Georgias del Sur y Sándwich del Sur. Sin embargo, cada Estado llevó a cabo sus estudios de manera independiente ${ }^{38}$.

El 21 de abril de 2009, Argentina notificó su presentación a la CLPC ${ }^{39}$ e informó de la existencia de un área controvertida respecto de las Islas Malvinas, Georgias del Sur e Islas Sándwich del Sur. En esta presentación, Argentina reivindicaba un largo margen continental que se extiende desde el Río de la Plata hasta el continente antártico, englobando los territorios de Malvinas, Georgias del Sur, Sándwich del Sur y el Sector Antártico Argentino.

Argentina definió el límite exterior de su plataforma continental aplicando las reglas anteriormente descritas. Una vez trazado el límite, se determinaron 79 puntos del pie del talud, utilizando en cada área el criterio más conveniente para asegurar la mayor extensión posible de la plataforma continental. Una vez determinados esos puntos del pie del talud, se aplicó la fórmula del 1\% del espesor sedimentario o de las 60 millas desde el pie del talud, según fuera conveniente, a fin de obtener los puntos fijos que determinarían el límite exterior de la plataforma continental argentina ${ }^{40}$. El área reivindicada ampliaría en más de $1.782 .000 \mathrm{~km}^{2}$ su plataforma continental más allá de las 200 millas marinas.

Diversos Estados ${ }^{41}$ objetaron esta presentación en lo relativo al Sector Antártico. La reacción de Reino Unido se hizo esperar tres meses ${ }^{42}$; y en su nota, además de impugnar la reclamación sobre el Ártico, rechazaba las reivindicaciones de soberanía de Argentina sobre los territorios en disputa y solicitaba a la CLPC que no examinase esas partes de la presentación.

Por su parte, Reino Unido notificó la ampliación de la plataforma continental proyectada por las Islas Malvinas, Georgias del Sur e Islas Sándwich del Sur el 11 de mayo de $2009^{43}$, con unos límites ligeramente diferentes a los de la propuesta argentina. El borde exterior de la plataforma continental se había calculado a lo largo de todo el trazado con la “fórmula Hedberg”. Para las líneas de restricción se empleaban ambas posibilidades.

\footnotetext{
${ }^{37}$ Canje de notas del 8 y 20 de junio.

38 DEL CASTILLO LABORDE, L.; La cuestión de las Islas Malvinas en el período 1983-2010, Publicación de la Asociación Profesional del Cuerpo Permanente del Servicio Exterior de la Nación Argentina, Año III Nº 3, 2010, pág. 39.

${ }^{39}$ CLCS.25.2009. LOS. (Continental Shelf Notification).

40 ARMAS PFIRTER, F.; El límite exterior de la plataforma continental. La presentación argentina y algunos aspectos de la práctica seguida hasta la fecha, Revista Jurídica de Buenos Aires, 2012, p. 222. Vid. ARMAS PFIRTER, F.; El Límite exterior de la plataforma continental argentina - el límite más extenso y nuestra frontera con la humanidad, Revista Res Diplomatica, n ${ }^{\circ}$ 1, 2015.

${ }^{41}$ Reino Unido, Estados Unidos, Rusia, India, Países Bajos y Japón.

42 Nota 84/09 de 6 de agosto de 2009. El intercambio de notas continúa, Argentina reafirmando sus derechos de soberanía (Nota 336/2012 de 8 de agosto de 2012) y Reino Unido manteniendo su posición (Nota 273/12 de 23 de agosto de 2012).

${ }^{43}$ CLCS.49.2009. LOS. (Continental Shelf Notification). 
Siguiendo la lógica anterior, Argentina rechazó de manera categórica la presentación británica y solicitó a la CLPC que ni la considerase ni la calificase ${ }^{44}$.

Las siguientes imágenes nos permiten apreciar las diferencias en el trazado que Argentina y Reino Unido realizaron del borde exterior de la plataforma continental y la subsiguiente línea envolvente de ampliación.

Argentina: Mapa con los puntos fijos del límite exterior de la plataforma continental argentina

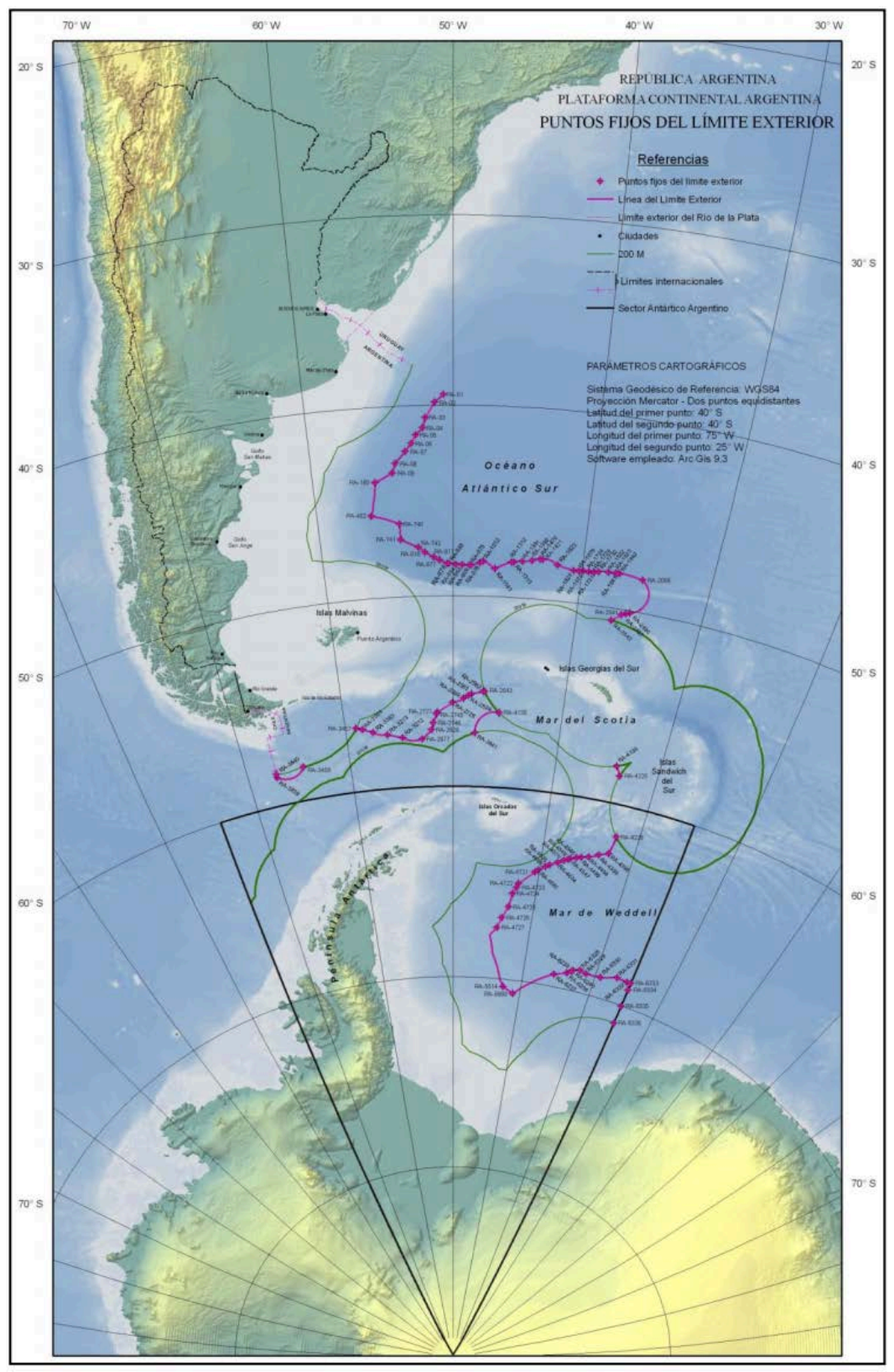

Fuente: Resumen ejecutivo de la presentación argentina a la CLPC.

${ }^{44}$ Nota 290/09/600 de 20 de agosto de 2009.

Revista de Estudios Jurídicos nº 17/2017 (Segunda Época) ISSN-e 2340-5066. Universidad de Jaén (España)

Versión electrónica: rej.ujaen.es 
Reino Unido: Límite exterior de la plataforma continental más allá de las 200 millas marinas para las Islas Malvinas, Georgias del Sur e Islas Sándwich del Sur

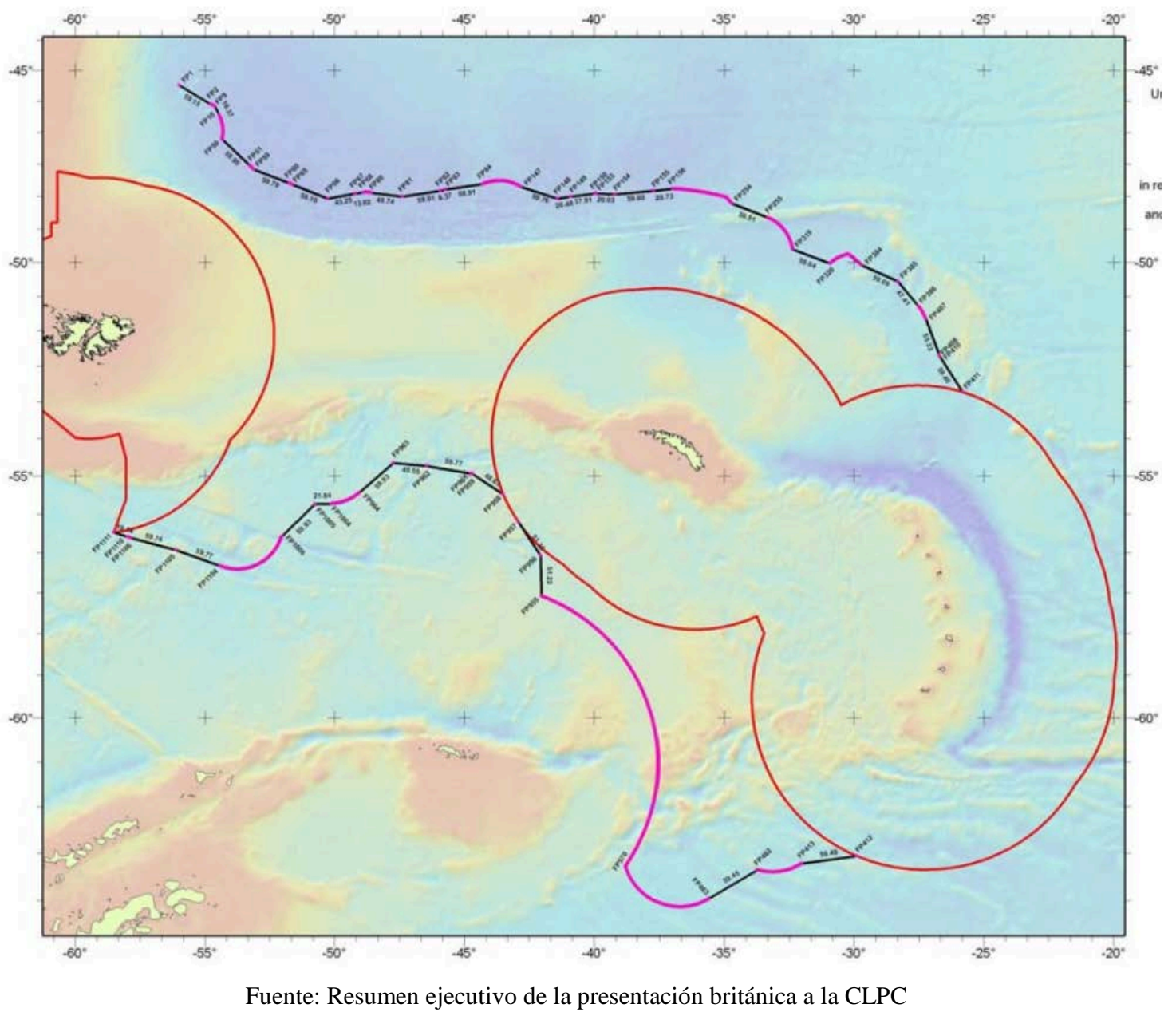

\section{EL TRATADO DE LA ANTÁRTIDA}

En 1959, los siete Estados ${ }^{45}$ que reivindicaban territorios de la Antártida congelaron sus demandas tras la firma del Tratado Antártico.

Este instrumento consagra la utilización de la Antártida para fines pacíficos y científicos y se aplica al continente antártico en la región situada al sur de los $60^{\circ}$ de latitud sur, incluidas todas las barreras de hielo, pero explícitamente no afecta a los derechos de los Estados respecto a la alta mar en esa zona.

Con la entrada en vigor de la Convención de Montego Bay, un nuevo sistema jurídico se incorpora a este panorama. La duda que surge es: ¿cómo se articula la existencia del Tratado Antártico y la Convención de Montego Bay?

\footnotetext{
${ }^{45}$ Argentina, Australia, Chile, Francia, Noruega, Nueva Zelanda y Reino Unido. Revista de Estudios Jurídicos no 17/2017 (Segunda Epoca) ISSN-e 2340-5066. Universidad de Jaén (España) Versión electrónica: rej.ujaen.es
} 
Recordemos que la Convención se funda en el principio "la tierra domina al mar", es por ello que el estatus de los todos los espacios marinos que instituye dependen del estatus del propio Estado ribereño. Sin embargo, en la Antártida no hay un Estado soberano que ejerza su jurisdicción.

Aunque el Tratado Antártico declara explícitamente que el Derecho del Mar se aplica a las aguas alrededor del continente, no dice nada sobre las zonas de jurisdicción extraterritorial. Esto deja abiertas cuestiones fundamentales sobre qué aspectos de la legislación oceánica contemporánea son relevantes para la Antártida y qué derechos y obligaciones marítimos son aplicables a cada Estado con intereses en la región ${ }^{46}$.

Se han generado dos posturas respecto a la posibilidad de ampliar la plataforma continental. Una parte de la doctrina defiende que la Convención de Montego Bay aplica una vez traspasado el paralelo $60^{\circ 47}$, mientras que otros estiman que la zona de ampliación debe quedar protegida por el régimen instaurado por el Tratado Antártico, puesto que involucra una extensión natural del continente o de las islas Antárticas ${ }^{48}$.

A pesar de las incertidumbres que rodean la aplicación de la Convención de Montego Bay y las disposiciones sobre la plataforma continental a la Antártida, seis de los siete Estados $^{49}$ que habían reclamado territorio en el continente blanco han realizado presentaciones ante la CLPC que afectan a la plataforma antártica.

Australia y Noruega presentaron a la Comisión las coordenadas de su margen continental, pero le pidieron que no las considerara por el momento ${ }^{50}$. Posteriormente,

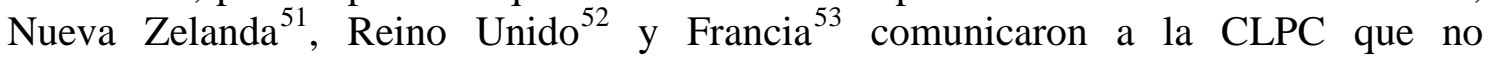
presentarían sus reclamaciones sobre la plataforma continental ampliada antártica, pero que se reservaban el derecho a hacerlo en un futuro.

\footnotetext{
${ }^{46}$ Vid. JOYNER, C.; Antarctica and the law of the sea, 1992, p. 75.

${ }^{47}$ Vid. PUCEIRO RIPOLL, R.; Los nuevos caminos jurídicos del sistema antártico: la Convención para la reglamentación de las actividades sobre recursos minerales antárticos, Revista de la Facultad de Derecho y Ciencias Sociales, Año XXIX, no 3-4, p. 388.

${ }^{48}$ Vid. ORREGO VICUÑA, F.; Derecho Internacional de la Antártida, Dolmen, 1994, p. 263.

${ }^{49}$ Además de Argentina, Australia presentó las coordenadas de su margen continental, pero pidió que la CLCS que no las considerara. Posteriormente, Nueva Zelanda, Reino Unido (presentación respecto a la Isla Ascensión) y Francia (presentación respecto a las Antillas Francesas y Kerguelen) comunicaron a la CLPC que no presentarían sus reclamaciones sobre la plataforma continental ampliada antártica, pero que se reservaban el derecho a hacerlo en un futuro. En la información preliminar presentada por Chile, este Estado menciona la Antártida como un área de posible extensión del límite exterior de su plataforma continental más allá de las 200 millas marinas. Chile declaró que la Comisión sería debidamente informada sobre la opción adoptada en relación con el "Territorio Antártico Chileno".

${ }^{50}$ Nota de 15 de noviembre de 2004 de la Misión Permanente de Australia ante el Secretario General de las Naciones Unidas que acompaña la notificación de su presentación; N89/2004 y Nota de 4 de mayo de 2009 de la Misión Permanente de Noruega ante el Secretario General de las Naciones Unidas que acompaña la notificación de su presentación sobre la Isla Bouvet y la Tierra de la Reina Maud.

${ }^{51}$ Nota de 19 de abril de 2006 de la Misión Permanente de Nueva Zelanda ante el Secretario General de las Naciones Unidas que acompaña la notificación de su presentación; NZ-CLCS-TPN-02.

${ }^{52}$ Nota de 9 de mayo de 2008 de la Misión Permanente de Reino Unido ante el Secretario General de las Naciones Unidas que acompaña la notificación de su presentación respecto de la Isla Ascensión; $\mathrm{N}^{\circ}$ $168 / 08$.

${ }^{53}$ Nota de 5 de mayo de 2009 de la Misión Permanente de Francia ante el Secretario General de las Naciones Unidas que acompaña la notificación de su presentación respecto de las Antillas Francesas y Kerguelen; Nota HR/cl N 69 .
} 
La postura de Argentina no pasa desapercibida ya que reclama la ampliación de la plataforma continental correspondiente al Sector Antártico Argentino. Argentina hizo notar a la CLPC que había tenido en cuenta las circunstancias del área ubicada al sur de los $60^{\circ}$ de latitud sur y el especial estatus legal y político de la Antártida a la luz de las disposiciones del Tratado Antártico ${ }^{54}$. Argentina justifica su postura aludiendo a que sus aspiraciones no afectan al territorio protegido por el tratado, sino a la ampliación de la plataforma continental que proyecta

Desde la Comisión se animó a que se optase por una presentación conjunta de todos los Estados con intereses en la Antártida ${ }^{55}$. Aun así, ninguna presentación a la CLCS, individual o conjunta, resolvería la cuestión primordial de la soberanía sobre la Antártida $^{56}$.

\section{LAS RECOMENDACIONES DE LA COMISIÓN DE LÍMITES DE LA PLATAFORMA CONTINENTAL}

El 11 de marzo de 2016, la Comisión emitió sus recomendaciones. En ellas se recoge un trazado de la plataforma continental argentina (en lo relativo a la parte continental) con mínimas variaciones respecto a la propuesta original relativas a la porción Norte del margen del Cratón del Río de la Plata, próximo al límite con Uruguay. Argentina realizó una presentación parcial revisada el 28 de octubre de 2016 atendiendo a las recomendaciones de la CLPC.

En relación con la ampliación de la plataforma continental de las Islas Malvinas, Georgias del Sur y Sándwich del Sur, la Comisión reconoce la existencia de una controversia relativa a la soberanía, por lo que decide no analizar por el momento el límite en esa área. Lo mismo ocurre con el Sector Antártico Argentino.

Resulta revelador que la Comisión no haya formado una Subcomisión para analizar la presentación de Reino Unido en relación con la plataforma continental de las Islas Malvinas, a raíz de la objeción presentada por la Argentina.

En definitiva, la Comisión posterga el análisis de los territorios cuya soberanía está en disputa. Al no existir un pronunciamiento al respecto en las recomendaciones, el trazado del límite de las Islas Malvinas, Georgias del Sur, Sándwich del Sur y del Sector Antártico no es oponible a terceros Estados, pues no puede ser considerado ni definitivo ni vinculante a nivel internacional.

\section{LA DELIMITACIÓN DE LA PLATAFORMA CONTINENTAL AMPLIADA Y SU RELACIÓN CON LA DETERMINACIÓN DEL LÍMITE EXTERIOR}

Al hilo de lo analizado en estas páginas respecto al trazado del límite exterior de la plataforma continental y su trascendencia de cara a su ampliación, no estaría de más

\footnotetext{
54 Nota de 21 de abril de 2009 de la Misión Permanente de la República Argentina ante el Secretario General de las Naciones Unidas que acompaña la notificación de su presentación; N.U.139/2009/600.

${ }^{55}$ Cita de las palabras de Alexandre Albuquerque, miembro de la CLPC, en el artículo de DOYLE, A.; Antarctica: the next North Pole? The International Herald Tribune, 1 de febrero de 2008. Accesible en: http://www.nytimes.com/2008/02/01/business/worldbusiness/01iht-green.4.9680882.html ${ }^{56}$ THORP, A.; Antarctica: the treaty system and territorial claims, International Affairs and Defence Section, Library House of Commons, 2012, p. 15. Vid. KAYE, S.; Antarctic maritime delimitations, en OUDE ELFERINK, A.; ROTHWELL, D.; The Law of the Sea and Polar Maritime Delimitation and Jurisdiction, Martinus Nijhoff, 2001, pág. 157-178.

Revista de Estudios Jurídicos nº 17/2017 (Segunda Época)

ISSN-e 2340-5066. Universidad de Jaén (España)

Versión electrónica: rej.ujaen.es
} 
entrar en la cuestión de la delimitación, es decir, de la determinación de la frontera marina que salva la confrontación entre Estados por la existencia de títulos superpuestos.

Es bien sabido que la CLPC debe quedarse al margen de las eventuales controversias de delimitación cuando emite sus recomendaciones. No obstante, la CLPC puede examinar una presentación que contenga una controversia territorial si los Estados manifiestan su consentimiento $^{57}$. Por lo tanto, la Comisión puede examinar las demandas de los Estados a pesar de la existencia de una frontera en disputa en la medida en la que la determinación de los límites no prejuzgue la delimitación.

Nos encontramos por tanto ante dos mecanismos distintos que difieren en su objetivo y en la institución encargada para darles solución. Por un lado, la determinación del límite exterior de la plataforma continental, y por ende del área de plataforma ampliada, en la que la CLPC juega el papel fundamental; por otro lado, la delimitación de la plataforma continental ampliada en caso de solapamiento, que se realizará mediante acuerdo entre los Estados con costas adyacentes u opuestas, y en caso de no alcanzarlo, acudiendo a la Parte XV de la Convención (solución de controversias) ${ }^{58}$.

Esta dualidad nos hace preguntarnos sobre la interacción entre ambos mecanismos: ¿qué ocurriría si un órgano jurisdiccional internacional se pronunciase sobre la delimitación de las plataformas continentales ampliadas adyacentes u opuestas antes de que la CLPC emita sus recomendaciones, o viceversa?

Para dar respuesta a esta cuestión debemos acudir a la jurisprudencia, y muy especialmente, a la solución otorgada por el Tribunal Internacional del Derecho del Mar en el asunto Bangladesh/Myanmar ${ }^{59}$ :

"Del mismo modo que las funciones de la Comisión no prejuzgan la cuestión de la delimitación de la plataforma continental entre Estados con costas opuestas o adyacentes, el ejercicio por las cortes y tribunales internacionales de su jurisdicción sobre la delimitación de las fronteras marítimas, no prejuzga el ejercicio por la Comisión de sus funciones en materia de delimitación de los límites exteriores de la plataforma continental”.

En definitiva, la CLPC podrá emitir sus recomendaciones en aquellos casos en los que un tribunal internacional haya delimitado previamente la plataforma continental ampliada y los órganos jurisdiccionales podrán pronunciarse sobre el trazado de una frontera marina, independientemente que existan unas recomendaciones de la Comisión.

\footnotetext{
${ }^{57}$ Exposición del Presidente de la CLPC sobre la labor de la Comisión, 17 de septiembre de 2010, 26 ${ }^{\mathrm{a}}$ sesión, CLCS/68, párrafo 51.

58 Aplicación analógica del artículo 83 de la Convención: “1. La delimitación de la plataforma continental entre Estados con costas adyacentes o situadas frente a frente se efectuará por acuerdo entre ellos sobre la base del derecho internacional, a que se hace referencia en el artículo 38 del Estatuto de la Corte Internacional de Justicia, a fin de llegar a una solución equitativa. 2. Si no se llegare a un acuerdo dentro de un plazo razonable, los Estados interesados recurrirán a los procedimientos previstos en la Parte XV".

59 Sentencia del TIDM de 14 de marzo de 2012 relativo a la Controversia sobre la Delimitación de las Fronteras Marítimas entre Bangladesh y Myanmar (Bangladesh contra Myanmar), caso nº 16, párrafo 379.
} 
Las futuras recomendaciones de CLPC para las presentaciones de Bangladesh y Myanmar podrán darnos más pistas al respecto.

¿Cómo afecta esta enunciación al caso que nos concierne? En la situación de impasse actual es poco probable que las recomendaciones de la Comisión se contrapongan con una más que hipotética sentencia. No obstante, ambos países podrían llegar a someter la controversia territorial (y la subsecuente delimitación marítima) a los tribunales internacionales.

\section{LA PLATAFORMA CONTINENTAL DE OTROS TERRITORIOS DISPUTADOS O NO AUTÓNOMOS}

Dada la dificultad que entraña el tratar con territorios cuya soberanía está cuestionada, acudamos a otros ejemplos de la escena internacional en busca de indicios que nos permitan arrojar algo de luz a este tipo de situaciones.

Revisando las presentaciones realizadas ante la CLPC encontramos algunos casos que en mayor o menor medida afectan a territorios disputados. Siguiendo el orden cronológico de las presentaciones, en primer lugar, aparece la presentación de Francia respecto a la Guayana Francesa y Nueva Caledonia ${ }^{60}$.

Nueva Caledonia es un archipiélago situado en el océano Pacífico Occidental y considerado como un territorio no autónomo por la Asamblea General de Naciones Unidas. Estas islas tienen estatus de "colectividad sui generis" pero es Francia la encargada de los asuntos exteriores, por lo que, a día de hoy, la legalidad de la presentación a la CLPC por parte de Francia es incuestionable.

Entre Nueva Caledonia y Vanuatu se encuentran las Islas Matthew y Hunter (incluidas en la presentación francesa), cuya soberanía también reivindica Vanuatu. Las objeciones realizadas por Vanuatu ${ }^{61}$ a la presentación de límites gala fueron acogidas por Francia, que solicitó a la Comisión que se abstuviera de pronunciarse sobre la plataforma continental del área cuestionada ${ }^{62}$.

Seguidamente, encontramos el desconcertante caso de la plataforma de Rockall, un peñón ubicado en el océano Atlántico Norte sujeto a soberanía británica. Este pequeño islote también ha sido reivindicado por Islandia, Dinamarca e Irlanda ${ }^{63}$. Todas las partes interesadas coinciden en que Rockall es una roca y que, por lo tanto, no proyecta una plataforma continental ${ }^{64}$.

La trascendencia de este asunto reside en que Reino Unido, Irlanda y Dinamarca ${ }^{65}$ han realizado presentaciones ante la CLPC relativas a los límites de su plataforma continental, y los tres Estados consideran como propio el peñón con el fin de utilizarlo como punto de base para el trazado del límite exterior de la plataforma continental. La Comisión aún no ha emitido sus recomendaciones.

\footnotetext{
${ }^{60}$ CLCS.08.2007.LOS (Continental Shelf Notification).

${ }^{61}$ Carta de 11 de julio de 2007.

${ }^{62}$ Nota $^{\circ}$ 547/SGMER de 18 de julio de 2007.

${ }^{63}$ Probablemente por las reservas de petróleo existentes en su plataforma continental.

64 Artículo 121.3 de la Convención de Montego Bay: "las rocas no aptas para mantener habitación humana o vida económica propia no tendrán zona económica exclusiva ni plataforma continental”

65 CLCS.19.2009.LOS (Continental Shelf Notification); CLCS.20.2009.LOS (Continental Shelf Notification); CLCS.54.2010.LOS (Continental Shelf Notification), respectivamente. Islandia se reservó su derecho a hacerlo en un futuro; Nota FNY09050022/97.B.512 de 27 de mayo de 2009. Revista de Estudios Jurídicos n ${ }^{\circ}$ 17/2017 (Segunda Época) ISSN-e 2340-5066. Universidad de Jaén (España) Versión electrónica: rej.ujaen.es
} 
El controvertido caso de las Islas Spratly y Paracelso también tiene implicaciones en la ampliación de la plataforma continental. En 2009, Vietnam y Malasia ${ }^{66}$ realizaron una presentación conjunta ante la Comisión en referencia a la parte sur del Mar de la China Meridional-, que abarcaba una pequeña parte de las Islas Spratly. En respuesta, China alegó su soberanía permanente sobre las islas de este $\operatorname{mar}^{67}$.

Vietnam realizó una presentación individual respecto a su zona norte ${ }^{68}$, que afecta a las Islas Paracelso y Spratly, y que también desencadenó la reacción de China y Filipinas. Posteriormente, China y Brunei ${ }^{69}$ realizaron presentaciones preliminares con un alto riesgo de solapamiento con las reivindicaciones de Vietnam, Malasia y Filipinas.

La CLPC no se ha pronunciado aún dada la controvertida soberanía. No obstante, cuenta con un nuevo elemento de análisis gracias al laudo de la Corte Permanente de Arbitraje de $2016^{70}$ en la disputa entre Filipinas y China.

Por último, se presenta el caso del territorio no autónomo de Tokelau, archipiélago situado en el Pacífico Sur que depende administrativamente de Nueva Zelanda.

En 2012, Tuvalu, Francia y Nueva Zelanda (Tokelau) realizaron una presentación conjunta a la CLPC ${ }^{71}$ relativa a la ampliación de la plataforma continental en la zona de Robbie Ridge. El hecho de que actualmente Nueva Zelanda actúe legítimamente al representar internacionalmente a Tokelau no aporta elementos que nos ayuden en nuestro análisis.

En definitiva, estos asuntos son una muestra más de cómo la CLPC se mantiene al margen cuando existe una controversia relativa a la soberanía. Los casos resueltos en los que un territorio no autónomo se ha visto involucrado no son comparables a la situación de Argentina y Reino Unido, pues el enfrentamiento no se produce entre dos Estados existentes por un territorio, sino entre la actual potencia que ejerce el poder y el pueblo autóctono con derecho de autodeterminación.

En otro orden de cosas, la jurisprudencia ha fallado en asuntos en los que se plantean al mismo tiempo la atribución del título de soberanía sobre un territorio y la delimitación marítima. En primer lugar, los tribunales resuelven las cuestiones de soberanía y, a la luz de la decisión, delimitan los espacios marinos entre los dos Estados.

En la sentencia de la Corte Internacional de Justicia en el asunto El Salvador contra Honduras $^{72}$, la Corte aprobó primero el trazado de la línea fronteriza en las secciones terrestres controvertidas entre El Salvador y Honduras. Seguidamente determinó la situación jurídica de las islas del Golfo de Fonseca, afirmando la soberanía hondureña

\footnotetext{
${ }^{66}$ CLCS.33.2009.LOS (Continental Shelf Notification).

67 Nota de 7 de mayo de 2009, CML/17/2009.

${ }^{68}$ CLCS.37.2009.LOS. (Continental Shelf Notification).

${ }^{69}$ Presentaciones preliminares de 11 y 12 de mayo de 2009, respectivamente.

${ }^{70}$ Laudo de la Corte Permanente de Arbitraje de 12 de julio de 2016 en el asunto Mar de la China Meridional (República de Filipinas contra República Popular China).

${ }^{71}$ CLCS.62.2012.LOS (Continental Shelf Notification).

${ }^{72}$ Sentencia de Corte Internacional de Justicia de 11 de septiembre de 1992 en el asunto relativo a la Controversia sobre fronteras terrestres, insulares y marítimas (El Salvador contra Honduras: intervención de Nicaragua).
} 
sobre El Tigre y la salvadoreña sobre Meanguera y Meanguerita. Finalmente, estableció los espacios marítimos situados dentro y fuera de la línea de cierre de ese Golfo.

En el asunto Catar contra Bahréin ${ }^{73}$, Catar incoó un procedimiento contra Bahréin ante la Corte Internacional de Justicia por una controversia relativa a la soberanía sobre una serie de islas, así como en materia de delimitación marítima entre ambos Estados.

La Corte examinó los antecedentes y situaciones de hecho de cada isla disputada y falló a favor de uno y otro Estado dependiendo del territorio en cuestión. A continuación, la Corte se ocupó de la delimitación.

En el caso de la controversia territorial y marítima entre Camerún contra Nigeria $^{74}$, Camerún sometió a la Corte Internacional de Justicia la cuestión relativa a la soberanía sobre la Península de Bakassi y solicitó una delimitación de las fronteras marítimas entre ambos Estados.

La Corte decidió que la soberanía sobre la Península de Bakassi recaía en Camerún y que la frontera ya estaba delimitada por un acuerdo anglo-alemán de 1913. Seguidamente procedió a delimitar los espacios marítimos.

Este caso guarda similitudes con la diferencia entre Colombia y Nicaragua, en la que la Corte Internacional de Justicia examinó la cuestión de la soberanía sobre una serie de formaciones insulares en disputa ${ }^{75}$. La Corte concluyó que no poseía jurisdicción para pronunciarse sobre la soberanía de las islas, debido a que la cuestión habría sido determinada por medio del Tratado sobre Cuestiones Territoriales Pendientes entre Colombia y Nicaragua de 1928, por medio del cual Nicaragua reconoció la soberanía colombiana sobre estas.

Una vez confirmada la autoridad colombiana, la Corte delimitó las respectivas plataformas continentales, ya que la plataforma continental que proyectan dichas islas se superpone con la plataforma nicaragüense.

No debe pasar inadvertido el hecho de que la Corte haya admitido una nueva demanda de Nicaragua para determinar el rumbo exacto de la plataforma continental entre las partes en el área más allá de las 200 millas marinas desde las líneas de base nicaragüenses ${ }^{76}$.

Terminaremos este análisis con la ya mencionada cuestión de las Islas Spratly. La Corte Permanente de Arbitraje, en un laudo de casi 500 páginas, no se pronuncia sobre cuestiones relacionadas con la soberanía o con la delimitación marítima, sino sobre la fuente de los derechos en el Mar de la China Meridional. La decisión declara que, a pesar de que China haya hecho uso de las islas, no se ha demostrado que hubiese ejercido históricamente el control exclusivo sobre las aguas o sus recursos, por lo tanto,

\footnotetext{
${ }^{73}$ Sentencia de la Corte Internacional de Justicia de 16 de marzo de 2001 en el asunto Delimitación marítima y cuestiones territoriales entre Catar y Bahréin.

${ }^{74}$ Sentencia de la Corte Internacional de Justicia de 10 de octubre de 2002 en el asunto relativo a las Fronteras terrestres y marítimas entre Camerún y Nigeria.

${ }^{75}$ Sentencia de la Corte Internacional de Justicia de 19 de noviembre de 2012 en el asunto Controversia territorial y marítima.

${ }^{76}$ Sentencia de la Corte Internacional de Justicia de 17 de marzo de 2016 en el asunto Cuestión de delimitación de la plataforma continental entre Nicaragua y Colombia más allá de las 200 millas marinas de la costa nicaragüense (Excepciones Preliminares). 
ni existe base legal para que China se atribuya la soberanía de las aguas de ese mar, ni se producen situaciones de solapamiento que necesiten de una delimitación posterior.

¿Qué lección sacamos de todo lo anterior? Es evidente que el sometimiento de una diferencia a tribunales internacionales entraña riesgos, pues una de las partes será el vencido en la disputa desde el momento en el que se adopte la decisión, y así será reconocido internacionalmente. En consecuencia, la posterior delimitación marítima también perjudicará a esa parte.

Si algún día Argentina y Reino Unido abandonasen la vía diplomática y decidiesen iniciar un procedimiento ante la jurisdicción internacional a propósito de las Islas Malvinas, Georgias del Sur y Sándwich del Sur, el escenario sería muy distinto dependiendo de los múltiples resultados.

Si un tribunal fallase a favor de Argentina, desaparecería la autoridad británica sobre las islas y no habría necesidad de delimitar, pues todo el espacio marítimo dependería de Buenos Aires. En el caso de que el órgano jurisdiccional decidiera a favor de Reino Unido, los jueces deberían trazar una línea de delimitación en el área de influencia de los archipiélagos. Podría también contemplarse el caso de que el tribunal atribuyese la soberanía de un archipiélago a una de las partes y la de los otros archipiélagos, a la parte contraria. Otra cuestión a tratar sería el estatus de las Islas Sándwich del Sur, que no están habitadas ni mantienen vida económica propia, y, por lo tanto, no proyectan plataforma continental.

\section{CONCLUSIÓN}

La ampliación de la plataforma continental es un procedimiento de extensión de derechos de soberanía deseable para la gran mayoría de los Estados ribereños. Gracias al progreso técnico, la plataforma continental ampliada se ha transformado en un espacio de una extraordinaria importancia geopolítica y geoeconómica en la que las naciones buscan nuevos recursos y tratan de afianzar su relevancia en la esfera internacional.

En la cuestión de la ampliación de la plataforma continental de las Islas Malvinas, Georgias del Sur y Sándwich del Sur, al afán de controlar nuevos recursos se une el anhelo por conseguir un reconocimiento como autoridad por parte de Argentina y Reino Unido. Ambos Estados reclaman su soberanía sobre estos territorios insulares y han incorporado la definición de los espacios marítimos a sus respectivos ordenamientos jurídicos.

La inclusión de los territorios en disputas en las presentaciones a la Comisión de Límites de la Plataforma Continental es una muestra más de unas pretensiones superpuestas. Sin embargo, la CLPC no es el órgano encargado de decidir sobre la soberanía de las islas, por lo que sus recomendaciones no pueden ser consideradas como el instrumento de arreglo definitivo de un conflicto.

Las recomendaciones emitidas por la Comisión para la presentación argentina reconocen la existencia de la controversia, algo que la Asamblea General de Naciones Unidas ya registró en 1965. Es un elemento más a favor de la autodeterminación de los 
pueblos, pero aún hoy, más de cincuenta años después, resulta difícil imaginar la solución para esta situación.

\section{BIBLIOGRAFÍA}

AMSTRONG, P.; Falklands Oil, IBRU Boundary and Security Bulletin, Durham University, julio 1994.

AMSTRONG P.; FORBES, V.; The Falkland Islands and their Adjacent Maritime Area, International Boundaries Research Unit, Maritime Briefing, Durham University, 1997.

ARMAS PFIRTER, F.; El límite exterior de la plataforma continental, Anuario Argentino de Derecho Internacional, X, Córdoba, Argentina, 2000.

ARMAS PFIRTER, F.; El límite exterior de la plataforma continental. La presentación argentina y algunos aspectos de la práctica seguida hasta la fecha, Revista Jurídica de Buenos Aires, 2012.

ARMAS PFIRTER, F.; El Límite exterior de la plataforma continental argentina - el límite más extenso y nuestra frontera con la humanidad, Revista Res Diplomatica, $\mathrm{n}^{\circ} 1$, 2015.

CANCLINI DE FIGUEROA, J.; JALABE, S.; Década de encuentro. Argentina y Gran Bretaña 1989-1999, Grupo Editor Latinoamericano, 2001.

DEL CASTILLO LABORDE, L.; La cuestión de las Islas Malvinas en el período 19832010, Publicación de la Asociación Profesional del Cuerpo Permanente del Servicio Exterior de la Nación Argentina, Año III N³, 2010.

DEL CASTILLO LABORDE, L.; Aspectos jurídicos de la cuestión Malvinas, en Islas Malvinas y Argentina 2006, Seminario organizado por el Instituto de Investigación y Desarrollo de la Academia Nacional de Ciencias de Buenos Aires, el 22 de junio de 2006.

DOYLE, A.; Antarctica: the next North Pole? The International Herald Tribune, 1 de febrero de 2008, recuperado el 20 de noviembre de 2016 en http://www.nytimes.com/2008/02/01/business/worldbusiness/01iht-

green.4.9680882.html

GUTIÉRREZ POSSE, H.; The significance and reasoning behind Dr. Jose Maria Ruda's arguments at the United Nations in relation to the question of sovereignty over the Malvinas (Falkland), South Georgia and South Sandwich Islands, en Liber Amicorum in memoriam of Judge José María Ruda, La Haya - Londres - Boston, Kluwer Law International, 2000.

FARAMIÑÁN GILBERT, J.M.; Situación actual del contencioso Malvinas (Falkland), Revista Española de Derecho Internacional, $\mathrm{n}^{0}$ 1-1985, Consejo Superior de Investigaciones Científicas, Instituto de Ciencias Jurídicas, Madrid, 1985.

JOYNER, C.; Antarctica and the law of the sea, 1992.

KAYE, S.; Antarctic maritime delimitations, en OUDE ELFERINK, A.; ROTHWELL, 
D.; The Law of the Sea and Polar Maritime Delimitation and Jurisdiction, Martinus Nijhoff, Leiden - Boston, 2001.

MOLENAAR, E.; OUDE ELFERINK, A.; ROTHWELL, D.; The Law of the Sea and the Polar Regions. Interactions between global and regional regimes, Martinus Nijhoff, Leiden - Boston, 2013.

ORREGO VICUÑA, F.; Derecho Internacional de la Antártida, Dolmen, 1994.

PUCEIRO RIPOLL, R.; Los nuevos caminos jurídicos del sistema antártico: la Convención para la reglamentación de las actividades sobre recursos minerales antárticos, Revista de la Facultad de Derecho y Ciencias Sociales, Año XXIX, nº 3-4.

SÁNCHEZ, R.; Antártida: Introducción a un continente remoto, Editorial Albatros, 2008.

THORP, A.; Antarctica: the treaty system and territorial claims, International Affairs and Defence Section, Library House of Commons, 2012. 